\title{
Prescription patterns and factors influencing the use of Chinese herbal medicine among pregnant women in
} Taiwan: a population-based retrospective study

\author{
Shu-Hui Wen ${ }^{1}$, Wei-Chuan Chang ${ }^{2}$, Hsuan-Shu Shen ${ }^{3,4}$ and Hsien-Chang Wu u, $^{4,}$ (D)
}

\begin{abstract}
Background: The use of Chinese herbal medicine (CHM) has been widely promoted as a natural and safe way to treat illness during pregnancy. However, prescription patterns and factors influencing its use are largely unknown. Therefore, we conducted a population-based study to address these questions.

Methods: Pregnant women aged 18-50 years were selected from Taiwan's National Health Insurance Research Database between 2001 to 2011. CHM prescriptions and diagnostic records were collected. Demographic data and pre-existing diseases were compared between CHM users and non-users. A multivariate logistic regression analysis was performed to identify possible factors influencing the use of CHM during pregnancy.

Results: A total of 81,873 eligible prescription records were identified, and 16,553 pregnant women were prescribed CHM during pregnancy, yielding a CHM prescription rate of $20.2 \%$. The three most frequently used herbs were Scutellariae Radix (Huang Qin) (4.4\%), Eucommiae cortex (Du Zhong) (2.5\%), and Atractylodes Rhizome (Bai Zhu) (2.4\%). The most frequently used herbal formulae were Dang-Guei-Shao-Yao-San (4.1\%), Jia-Wei-Xiao-Yao-San (3.5\%), and Xiang-Sha-Liu-Jun-Zi-Tang (2.6\%). Multivariate logistic regression revealed that subjects with an older age, a university education, a pre-pregnancy history of $\mathrm{CHM}$ use, asthma, chronic renal disease, and cardiac valvular disease and living in a residential area other than northern Taiwan had an increase in adjusted odds ratio for CHM use during pregnancy.

Conclusions: In this population-based study, we found that demographic factors and pre-existing diseases were associated with the use of CHM among pregnant women. It is worth noting that Leonuri Herba (Yi Mu Cao) and ShaoFu-Zhu-Yu-Tang should be used with caution in the first trimester. Further research is needed to explore the safety and effectiveness of the use of CHM in pregnant women.
\end{abstract}

Keywords: Alternative medicine, Chinese herbal medicine, Pregnant women, Peripartum, Perinatal, Traditional Chinese medicine

\footnotetext{
* Correspondence: dr30032@gmail.com

${ }^{4}$ School of Post-baccalaureate Chinese Medicine, Tzu Chi University, Hualien, Taiwan

${ }^{5}$ Department of Chinese Medicine, Taipei Tzu Chi Hospital, The Buddhist Tzu Chi Medical Foundation, No. 289, Jianguo Rd., Xindian Dist, New Taipei City 23142, Taiwan

Full list of author information is available at the end of the article
}

(c) The Author(s). 2020 Open Access This article is licensed under a Creative Commons Attribution 4.0 International License, which permits use, sharing, adaptation, distribution and reproduction in any medium or format, as long as you give appropriate credit to the original author(s) and the source, provide a link to the Creative Commons licence, and indicate if changes were made. The images or other third party material in this article are included in the article's Creative Commons licence, unless indicated otherwise in a credit line to the material. If material is not included in the article's Creative Commons licence and your intended use is not permitted by statutory regulation or exceeds the permitted use, you will need to obtain permission directly from the copyright holder. To view a copy of this licence, visit http://creativecommons.org/licenses/by/4.0/ The Creative Commons Public Domain Dedication waiver (http://creativecommons.org/publicdomain/zero/1.0/) applies to the data made available in this article, unless otherwise stated in a credit line to the data. 


\section{Background}

Chinese herbal medicine (CHM) has been widely used as a natural and safe way to treat illness during pregnancy [1]. The reported prevalence of medicinal herb usage during pregnancy ranges from 12 to $58 \%$ in various countries [2-6]. In Taiwan, 34\% of Taiwanese women consume at least one type of CHM during pregnancy [5]. In clinical settings, women tend to take $\mathrm{CHM}$ as an alternative treatment for a variety of pregnancy diseases, such as vomiting during pregnancy, hypertension, and miscarriage $[7,8]$. However, the prescription patterns and factors influencing the use of CHM during pregnancy are largely unknown.

Previous studies have shown that there are differences in the socio-demographic and health-related characteristics between users of complementary and alternative medicine (CAM) and non-users [9]. For example, subjects with old age, females, high socioeconomic groups, and those with a high education level and health problems are prone to use CAM in countries other than Taiwan [9-11]. Similar findings have also been reported by investigators who used the National Health Insurance Research Database (NHIRD) in Taiwan [12, 13]. However, factors influencing the use of CHM in the subset of pregnant women remain unclear.

Taiwan's health authority maintains traditional Chinese medicine (TCM) under the auspices of the National Health Insurance (NHI) Service and enhances CHM quality control management to ensure the health and medical care for the public. NHIRD was established by the NHI as a universal health insurance program since 1995 in Taiwan. The database covers more than $99.6 \%$ of the Taiwanese population $[14,15]$. Particularly, pregnant women who need CHM treatment are required to obtain prescriptions from TCM physicians. Thus, NHIRD is a large database that comprehensively includes demographic and clinical data of all pregnant women who receive TCM. An understanding of the prescription patterns may be helpful for monitoring and evaluating the adequacy of CHM usage among pregnant women. In addition, an analysis of the factors related to $\mathrm{CHM}$ use may provide essential information to clinicians regarding the profiles of pregnant women who are more likely to use CHM. In this study, we aimed to investigate the prescription patterns and factors influencing the use of $\mathrm{CHM}$ during pregnancy by using this dataset. To achieve this goal, the prescription patterns in relation to diagnoses were evaluated. Factors influencing the use of CHM were identified by comparisons of variables between the CHM users and non-users.

\section{Methods}

\section{Data resources}

We conducted a retrospective population-based study using the longitudinal health insurance database (LHID) for the period from 2001 to 2011, which was provided by the Health and Welfare Statistics Application Centre of the Ministry of Health and Welfare in Taiwan. The LHID comprises medical claims data of more than 2 million beneficiaries who have been randomly sampled since the year 2000 from registries of all NHI enrolees. The LHID contains data on patient demographic characteristics, outpatient and inpatient visit records, drug prescriptions (including $\mathrm{CHM}$ ), and International Classification of Disease-Clinical Modification (9th revision) (ICD-9-CM) diagnoses. The information on diagnosis codes of the database have been validated by a previous study [16]. This study was approved by the Institutional Review Board of Taipei Tzu Chi Hospital in Taipei, Taiwan (Protocol No. 05-W03-050).

\section{Study sample and measured variables}

Medical records of pregnant women were selected from the LHID for the period between 2001 and 2011 using the following inclusion criteria: (1) aged between 18 and 50 years; (2) confirmed as pregnant (ICD-9-CM codes: V22 and V23) via outpatient or inpatient visit; and (3) a birth outcome of term birth (ICD-9-CM codes: V27.0, V27.2, V27.3, V27.5, V27.6, V30-V37, V39, 650), preterm birth (ICD-9-CM code: 765.1), abortion (ICD-9-CM codes: 632; ICD_OP_CODE: 690, 695, 750), or stillbirth (ICD-9-CM codes: V27.1, V27.4, V27.7, 656.4). We estimated the start date of pregnancy according to the initial prenatal visit which represents its specific timing during pregnancy. The duration of pregnancy was defined as the time between the start date of pregnancy and the date of the end of pregnancy due to a live birth, abortion, or stillbirth. We excluded cases based on the following criteria: male gender, no record of birth outcome, age outside of the specified inclusion range, a pre-pregnancy malignant tumour history, or incomplete prenatal visits. A total of 81,873 eligible pregnant women were identified. Figure 1 shows the flowchart of the study.

Among these, 16,553 pregnant women (20.2\%) used $\mathrm{CHM}$ which was determined by correlating TCM outpatient visits and $\mathrm{CHM}$ prescription records. All CHM prescription records were classified as either single herbs or herbal formulae. Prescription patterns and primary diagnostic codes associated with TCM outpatient visits were presented in terms of three (first, second, third) trimester groups. To understand the prescription patterns and safety of $\mathrm{CHM}$ use during human embryogenesis (6-12 weeks of gestation), we analysed all gynaecological diagnoses and prescriptions recorded both before and after the first prenatal visit. That is, we assessed common diagnostic codes and CHM use before or after the first prenatal visit only among women with obstetricsrelated TCM records (ICD-9-CM code: $610-677$ or A code: A370-A394). Women may have consulted with 
their TCM physicians prior to a confirmed pregnancy, before their first prenatal visit. Thus, it was important to assess CHM use during this period.

Sample characteristics, including age at pregnancy, education level (below junior, senior, university, or above), income (monthly income in new Taiwan dollars [NTD]), residential area (northern, middle, southern, or eastern Taiwan), prior usage (whether she had used CHM within the 6 months before pregnancy), and maternal comorbidity, were also collected. Maternal comorbidities were diagnosed with ICD-9-CM codes (Supplementary Table 2) from at least one ambulatory visit or inpatient claim within the year prior to pregnancy [17].

\section{Statistical analyses}

Descriptive statistics (numbers and percentages) were provided for the distribution of primary diagnostic codes and CHM use. Outpatient TCM records from pregnancy were further restricted to obstetrics-related codes. The 10 most common primary diagnostic codes and CHMs were presented for the three trimesters, as well as before or after the first prenatal visit. A logistic regression analysis was performed to examine the factors associated with $\mathrm{CHM}$ use during pregnancy. The covariates were age at pregnancy, education level, income, residential area, prior usage, and maternal comorbidity. Odds ratios (ORs) and accompanying 95\% confidence intervals (CIs) were provided. Data analyses were performed with SAS version 9.3 (SAS Institute, Inc., Cary, NC, USA).

\section{Results}

Sample characteristics

A total of 16,553 pregnant women received CHM during pregnancy (termed 'CHM users' hereafter), yielding a CHM utilization rate of $20.2 \%$. The average duration between the estimated start date of pregnancy and the first CHM prescription record was 62.8 days. In total, $81.7 \%$ of the women received their first CHM prescription during the first trimester. The average duration of $\mathrm{CHM}$ use was 19.8 days during pregnancy.

Some characteristics differed significantly between CHM users and non-users (Table 1). CHM users were slightly older, had longer pregnancies, and had higher education and income levels $(P<0.05)$. More $\mathrm{CHM}$ users lived in middle and southern Taiwan than non-users. Slightly higher percentages of maternal comorbidities such as asthma, chronic renal disease, Sickle cell disease, and cardiac valvular disease were present in CHM users compared to non-users $(P<0.05)$. Finally, CHM use 6 months prior to pregnancy was more common among TCM users than non-users $(63.1 \%$ vs. $22.7 \%, P<0.05)$.

\section{Prescription patterns of CHM users}

The top 10 single herbs, herbal formulae, and primary diagnostic codes identified among CHM users are listed in Supplementary Table 1. For all CHM records, the top three most common primary diagnostic codes were abnormal bleeding from the female genital tract (12\%), acute nasopharyngitis (11.2\%), and cough (5.2\%). The most commonly used single herbs were Scutellariae

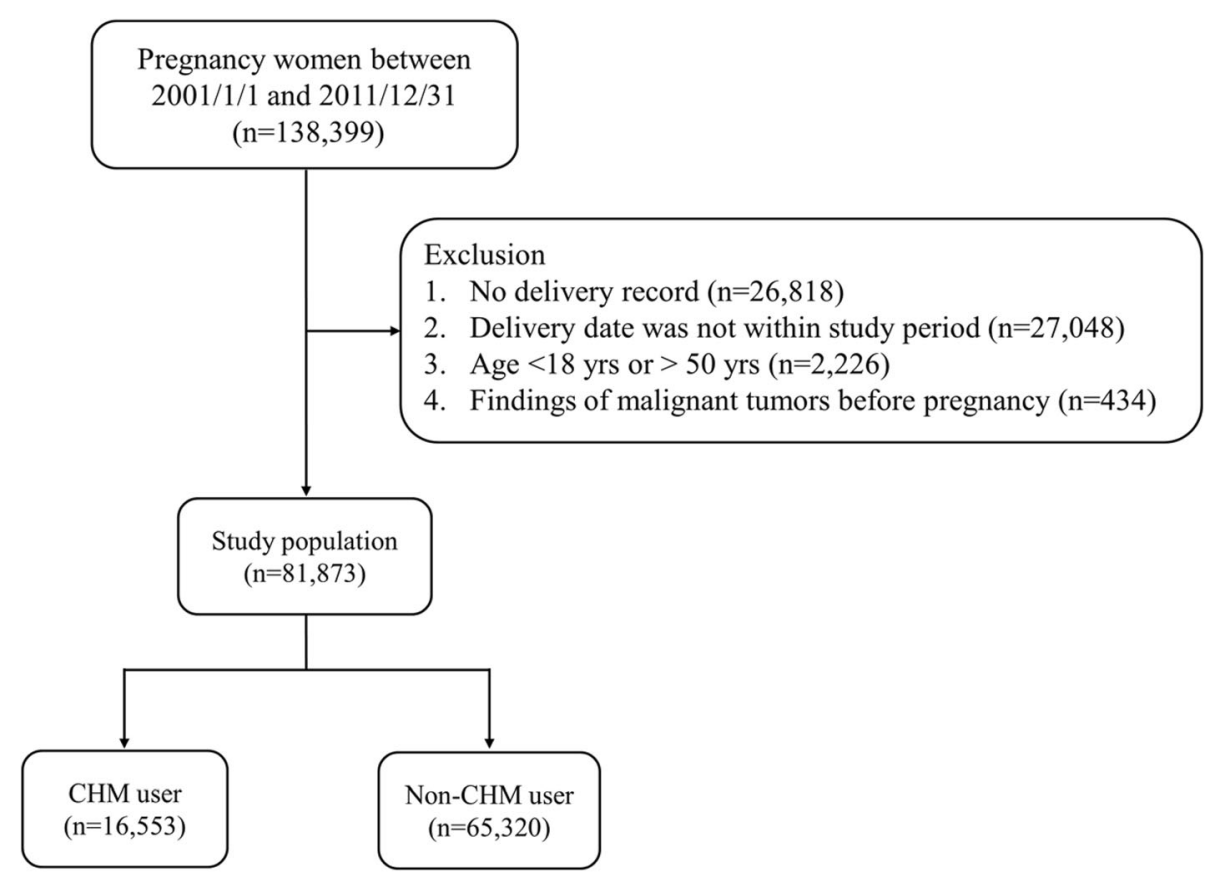

Fig. 1 Flow chart of the study population 
Radix (Huang Qin) (4.4\%), Eucommiae cortex (Du Zhong) (2.5\%), Atractylodes Rhizome (Bai Zhu) (2.4\%), Cyperi Rhizoma (Xiang Fu) (2.1\%), Cuscutae Semen (Tu $\mathrm{Si} \mathrm{Zi})$ (2\%), and Dipsaci Radix (Xu Duan) (2\%). The most commonly used herbal formulae were Dang-Guei-ShaoYao-San (4.1\%), Jia-Wei-Xiao-Yao-San (3.5\%), XiangSha-Liu-Jun-Zi-Tang (2.6\%), Bao-Chan-Wu-You-Fang (2.6\%), Yin-Qiao-San (2.2\%), and Xin-Yi-Qing-Fei-Tang (2\%), which together accounted for $17 \%$ of the herbal formulae prescribed.

There were also differences identified among the three trimester groups regarding diagnostic codes and prescription patterns (Table 2). For instance, the most common diagnostic code issued in the first trimester was abnormal bleeding from the female genital tract (17.2\%), while acute nasopharyngitis was most common during the second (11.9\%) and third
(16.5\%) trimesters. Hypertension complicating pregnancy, childbirth, and the puerperium (6.9\%) and sleep disturbances (3.1\%) became more common in the third trimester. Increased use of Atractylodes Rhizome (Bai Zhu) and Platycodi Radix (Jie Geng) was observed in the second and third trimesters. In particular, single herbs such as Leonuri Herba (Yi $\mathrm{Mu} \mathrm{Cao}$ ) and Corydalis Rhizoma (Yan $\mathrm{Hu} \mathrm{Su}$ ) and herbal formulae such as Wen-Jing-Tang and BanXia-Xie-Xin-Tang were used only during the first trimester.

We further limited our assessments to obstetricsrelated records and CHM usage either before or after the first prenatal visit (Table 3 ). We found that abnormal bleeding from the female genital tract (53\%), infertility (10.4\%), and dysmenorrhea (8.3\%) were the three most common primary diagnoses encountered

Table 1 Characteristics between pregnant women with and without CHM use ( $n=81,873)$

\begin{tabular}{|c|c|c|c|}
\hline Characteristics & $\begin{array}{l}\text { CHM user } \\
n=16,553\end{array}$ & $\begin{array}{l}\text { Non-CHM user } \\
n=65,320\end{array}$ & $p$-value \\
\hline Age (years) & $28.6 \pm 4.6$ & $28.0 \pm 4.8$ & $<0.001$ \\
\hline Duration of pregnancy (days) & $266.3 \pm 63.5$ & $255.9 \pm 69.8$ & $<0.001$ \\
\hline Education & & & $<0.001$ \\
\hline Below Junior & $3582(22.1)$ & $16,203(25.5)$ & \\
\hline Senior & $7148(44.1)$ & 28,521 (44.8) & \\
\hline University or above & 5489 (33.8) & $18,912(29.7)$ & \\
\hline Income level (1000 NTD per unit) & $24.7 \pm 16.2$ & $23.9 \pm 16.9$ & $<0.001$ \\
\hline$<20,000$ & $5858(41.7)$ & $24,189(44.5)$ & $<0.001$ \\
\hline $20,000-39,999$ & $5685(40.4)$ & $21,031(38.7)$ & \\
\hline$>40,000$ & $2512(17.9)$ & $9147(16.8)$ & \\
\hline Residential area & & & $<0.001$ \\
\hline Northern & $5565(33.7)$ & $28,861(44.3)$ & \\
\hline Middle & $4621(27.9)$ & $11,890(18.3)$ & \\
\hline Southern & 5538 (33.6) & $20,467(31.4)$ & \\
\hline Eastern & $784(4.8)$ & $3916(6.0)$ & \\
\hline \multicolumn{4}{|l|}{ Maternal Comorbidity } \\
\hline Asthma & $352(2.1)$ & $1036(1.6)$ & $<0.001$ \\
\hline Chronic ischemic heart disease & $50(0.3)$ & $149(0.2)$ & 0.084 \\
\hline Chronic renal disease & $119(0.7)$ & $256(0.4)$ & $<0.001$ \\
\hline Congenital heart disease & $13(0.1)$ & $52(0.1)$ & 0.965 \\
\hline Preexisting diabetes mellitus & $118(0.7)$ & $402(0.6)$ & 0.159 \\
\hline Preexisting hypertension & $77(0.5)$ & $303(0.5)$ & 0.982 \\
\hline Sickle cell disease & $37(0.2)$ & $88(0.1)$ & 0.009 \\
\hline Cardiac valvular disease & $189(1.1)$ & $441(0.7)$ & $<0.001$ \\
\hline Placenta previa & $6(0.1)$ & $36(0.1)$ & 0.338 \\
\hline Gestational hypertension & $12(0.1)$ & $33(0.1)$ & 0.281 \\
\hline CHM use at 6 months before first pregnancy diagnosis & $10,449(63.1)$ & $14,812(22.7)$ & $<0.001$ \\
\hline
\end{tabular}

Data are shown as $\mathrm{n}(\%)$, or mean \pm standard deviation CHM Chinese herbal medicine, NTD New Taiwan dollar 
Table 2 Top 10 single herbal, Herbal formulae and diagnosis and prescription in CHM users at three trimesters

\begin{tabular}{|c|c|c|c|c|c|}
\hline \multicolumn{2}{|l|}{ First trimester } & \multicolumn{2}{|l|}{ Second trimester } & \multicolumn{2}{|l|}{ Third trimester } \\
\hline & $\begin{array}{l}\text { Frequency } \\
(\%)\end{array}$ & & $\begin{array}{l}\text { Frequency } \\
(\%)\end{array}$ & & $\begin{array}{l}\text { Frequency } \\
\text { (\%) }\end{array}$ \\
\hline \multicolumn{6}{|l|}{ Diagnosis } \\
\hline $\begin{array}{l}\text { Abnormal bleeding from } \\
\text { female genital tract }\end{array}$ & $\begin{array}{l}34,960 \\
(17.2)\end{array}$ & Acute nasopharyngitis & $9311(11.9)$ & Acute nasopharyngitis & $6801(16.5)$ \\
\hline Acute nasopharyngitis & $\begin{array}{l}18,080 \\
(8.9)\end{array}$ & $\begin{array}{l}\text { Abnormal bleeding from female } \\
\text { genital tract }\end{array}$ & $8467(10.8)$ & Cough & $3618(8.8)$ \\
\hline $\begin{array}{l}\text { Disorders of function of } \\
\text { stomach }\end{array}$ & $9446(4.7)$ & Cough & $4940(6.3)$ & $\begin{array}{l}\text { Hypertension complicating pregnancy, } \\
\text { childbirth, and the puerperium }\end{array}$ & $2846(6.9)$ \\
\hline $\begin{array}{l}\text { Excessive vomiting in } \\
\text { pregnancy }\end{array}$ & $7445(3.7)$ & Headache & $3102(4.0)$ & Disorders of function of stomach & $1826(4.4)$ \\
\hline Headache & $7370(3.6)$ & $\begin{array}{l}\text { Hypertension complicating pregnancy, } \\
\text { childbirth, and the puerperium }\end{array}$ & $3023(3.9)$ & Sleep disturbances & $1279(3.1)$ \\
\hline Constipation & 7339 (3.6) & Sleep disturbances & $2909(3.7)$ & Headache & $1228(3.0)$ \\
\hline Cough & $7177(3.5)$ & Disorders of function of stomach & $2885(3.7)$ & Low back pain & $1191(2.9)$ \\
\hline Sleep disturbances & $6836(3.4)$ & Constipation & $2594(3.3)$ & Constipation & $1191(2.9)$ \\
\hline $\begin{array}{l}\text { Female infertility } \\
\text { associated with } \\
\text { anovulation }\end{array}$ & $6715(3.3)$ & Allergic rhinitis & $2337(3.0)$ & Pruritus and related conditions & $1188(2.9)$ \\
\hline Dysmenorrhea & $5432(2.7)$ & Excessive vomiting in pregnancy & $2214(2.8)$ & $\begin{array}{l}\text { Chronic pharyngitis and } \\
\text { nasopharyngitis }\end{array}$ & $1137(2.7)$ \\
\hline \multicolumn{6}{|l|}{ Single herbal } \\
\hline $\begin{array}{l}\text { Scutellariae Radix (Huang } \\
\text { Qin) }\end{array}$ & $4023(3.5)$ & Scutellariae Radix (Huang Qin) & $2096(4.7)$ & Scutellariae Radix (Huang Qin) & $1504(6.3)$ \\
\hline Cyperi Rhizoma (Xiang Fu) & $3191(2.7)$ & Eucommiae cortex (Du Zhong) & $1288(2.9)$ & Atractylodes Rhizome (Bai Zhu) & $845(3.5)$ \\
\hline $\begin{array}{l}\text { Eucommiae cortex (Du } \\
\text { Zhong) }\end{array}$ & $2894(2.5)$ & Atractylodes Rhizome (Bai Zhu) & $1133(2.5)$ & Eucommiae cortex (Du Zhong) & $679(2.8)$ \\
\hline Cuscutae Semen (Tu Si Zi) & $2740(2.4)$ & Dipsaci Radix (Xu Duan) & $961(2.1)$ & Platycodi Radix (Jie Geng) & $610(2.5)$ \\
\hline $\begin{array}{l}\text { Corydalis Rhizoma (Yan Hu } \\
\text { Su) }\end{array}$ & $2426(2.1)$ & Platycodi Radix (Jie Geng) & $864(1.9)$ & Fritillariae Thunbergii Bulbus (Bei Mu) & $549(2.3)$ \\
\hline Leonuri Herba (Yi Mu Cao) & $2353(2.0)$ & Cyperi Rhizoma (Xiang Fu) & $843(1.9)$ & Dipsaci Radix (Xu Duan) & $536(2.2)$ \\
\hline Dipsaci Radix (Xu Duan) & $2280(1.9)$ & Cuscutae Semen (Tu Si Zi) & $826(1.8)$ & $\begin{array}{l}\text { Glycyrrhizae Radix et Rhizoma (Gan } \\
\text { Cao) }\end{array}$ & $446(1.9)$ \\
\hline $\begin{array}{l}\text { Atractylodes Rhizome (Bai } \\
\text { Zhu) }\end{array}$ & $2154(1.8)$ & Fritillariae Thunbergii Bulbus (Bei Mu) & $690(1.5)$ & Ophiopogonis Radix (Mai Men Dong) & $430(1.8)$ \\
\hline $\begin{array}{l}\text { Rhei Radix et Rhizoma (Da } \\
\text { Huang) }\end{array}$ & $1840(1.6)$ & $\begin{array}{l}\text { Glycyrrhizae Radix et Rhizoma (Gan } \\
\text { (ao) }\end{array}$ & $683(1.5)$ & Taxilli Herba (Sang Ji Sheng) & $407(1.7)$ \\
\hline Platycodi Radix (Jie Geng) & $1670(1.4)$ & Ophiopogonis Radix (Mai Men Dong) & $680(1.5)$ & Scrophulariae Radix (Xuan Shen) & $371(1.6)$ \\
\hline \multicolumn{6}{|l|}{ Herbal formulae } \\
\hline Dang-Gui-Shao-Yao-San & $3805(4.4)$ & Dang-Gui-Shao-Yao-San & $1447(4.3)$ & Bao-Chan-Wu-You-Fang & $898(5.2)$ \\
\hline Jia-Wei-Xiao-Yao-San & $3781(4.3)$ & Jia-Wei-Xiao-Yao-San & $1047(3.1)$ & Dang-Gui-Shao-Yao-San & $648(3.7)$ \\
\hline Xiang-Sha-Liu-Jun-Zi-Tang & $2240(2.6)$ & Bao-Chan-Wu-You-Fang & $823(2.5)$ & Yin-Qiao-San & $599(3.4)$ \\
\hline Wen-Jing-Tang & $1836(2.1)$ & Yin-Qiao-San & $749(2.2)$ & Xin-Yi-Qing-Fei-Tang & $517(3.0)$ \\
\hline Ban-Xia-Xie-Xin-Tang & $1601(1.8)$ & Xin-Yi-Qing-Fei-Tang & $711(2.1)$ & Ma-Xing-Gan-Shi-Tang & $403(2.3)$ \\
\hline Ping-Wei-San & $1530(1.8)$ & Xiang-Sha-Liu-Jun-Zi-Tang & $677(2.0)$ & Xiang-Sha-Liu-Jun-Zi-Tang & $370(2.1)$ \\
\hline Yin-Qiao-San & $1529(1.8)$ & Chuan-Qiong-Cha-Tiao-San & $622(1.9)$ & Gan-Lu-Yin & $366(2.1)$ \\
\hline Gui-Zhi-Fu-Ling-Wan & $1521(1.7)$ & Gui-Pi-Tang & $557(1.7)$ & Sang-Ju-Yin & $342(2.0)$ \\
\hline $\begin{array}{l}\text { Chuan-Qiong-Cha-Tiao- } \\
\text { San }\end{array}$ & $1473(1.7)$ & Ma-Zi-Ren-Wan & $541(1.6)$ & Xiao-Chai-Hu-Tang & 335 (1.9) \\
\hline Ma-Zi-Ren-Wan & $1430(1.6)$ & Ping-Wei-San & $534(1.6)$ & Jia-Wei-Xiao-Yao-San & $327(1.9)$ \\
\hline
\end{tabular}


Table 3 Top 10 obstetrics related diagnosis and prescription in pregnancy women before and after the first prenatal visit

\begin{tabular}{|c|c|c|c|c|}
\hline & Before prenatal Visit & & After prenatal Visit & \\
\hline \multirow[t]{10}{*}{$\begin{array}{l}\text { Diagnosis } \\
n(\%)^{a}\end{array}$} & Abnormal bleeding from female genital tract & $\begin{array}{l}28,738 \\
(53.0)\end{array}$ & Abnormal bleeding from female genital tract & $\begin{array}{l}55,460 \\
(53.3)\end{array}$ \\
\hline & Infertility & $\begin{array}{l}5649 \\
(10.4)\end{array}$ & Dysmenorrhea & $9403(9.0)$ \\
\hline & Dysmenorrhea & $4494(8.3)$ & $\begin{array}{l}\text { Hypertension complicating pregnancy, Childbirth, and } \\
\text { the puerperium }\end{array}$ & $8014(7.7)$ \\
\hline & Excessive vomiting in pregnancy & $4087(7.5)$ & Excessive vomiting in pregnancy & $6729(6.5)$ \\
\hline & $\begin{array}{l}\text { Hypertension complicating pregnancy, Childbirth, and } \\
\text { the puerperium }\end{array}$ & $2843(5.2)$ & Infertility & $6364(6.1)$ \\
\hline & Leukorrhea & $2131(3.9)$ & Leukorrhea & $5311(5.1)$ \\
\hline & Hemorrhage in early pregnancy & $1213(2.2)$ & Other complications of pregnancy & $2350(2.3)$ \\
\hline & Endometriosis & $905(1.7)$ & Hemorrhage in early pregnancy & $1525(1.5)$ \\
\hline & Premenstrual tension syndromes & $885(1.6)$ & Premenstrual tension syndromes & $1284(1.2)$ \\
\hline & Other complications of pregnancy & $622(1.1)$ & Endometriosis & $963(0.9)$ \\
\hline \multirow{10}{*}{$\begin{array}{l}\text { Single herbal } \\
n(\%)^{b}\end{array}$} & Cyperi Rhizoma (Xiang Fu) & $1724(5.0)$ & Cyperi Rhizoma (Xiang Fu) & $3109(4.7)$ \\
\hline & Cuscutae Semen (Tu Si Zi) & $1555(4.5)$ & Leonuri Herba (Yi Mu Cao) & $2686(4.1)$ \\
\hline & Leonuri Herba (Yi Mu Cao) & $1450(4.2)$ & Corydalis Rhizoma (Yan Hu Su) & $2104(3.2)$ \\
\hline & Eucommiae cortex (Du Zhong) & $1161(3.4)$ & Eucommiae cortex (Du Zhong) & $2027(3.1)$ \\
\hline & Scutellariae Radix (Huang Qin) & $1090(3.2)$ & Cuscutae Semen (Tu Si Zi) & $1986(3.0)$ \\
\hline & Corydalis Rhizoma (Yan Hu Su) & $943(2.7)$ & Scutellariae Radix (Huang Qin) & $1946(3.0)$ \\
\hline & Dipsaci Radix (Xu Duan) & $927(2.7)$ & Salviae Miltiorrhizae Radix ET Rhizoma (Dan Shen) & $1479(2.3)$ \\
\hline & Salviae Miltiorrhizae Radix ET Rhizoma (Dan Shen) & $720(2.1)$ & Dipsaci Radix (Xu Duan) & $1439(2.2)$ \\
\hline & Atractylodes Rhizome (Bai Zhu) & $681(2.0)$ & Atractylodes Rhizome (Bai Zhu) & $1207(1.8)$ \\
\hline & Ligustri Lucidi Fructus (Nu Zhen Zi) & $598(1.7)$ & Rhei Radix et Rhizoma (Da Huang) & $924(1.4)$ \\
\hline \multirow{10}{*}{$\begin{array}{l}\text { Herbal } \\
\text { formulae } \\
n(\%)^{c}\end{array}$} & Dang-Gui-Shao-Yao-San & $2082(8.9)$ & Jia-Wei-Xiao-Yao-San & $3599(7.9)$ \\
\hline & Jia-Wei-Xiao-Yao-San & $1844(7.9)$ & Dang-Gui-Shao-Yao-San & $3492(7.7)$ \\
\hline & Wen-Jing-Tang & $1294(5.5)$ & Wen-Jing-Tang & $2213(4.9)$ \\
\hline & Gui-Zhi-Fu-Ling-Wan & $896(3.8)$ & Gui-Zhi-Fu-Ling-Wan & $1953(4.3)$ \\
\hline & Shao-Fu-Zhu-Yu-Tang & $602(2.6)$ & Gui-Pi-Tang & $1292(2.8)$ \\
\hline & Gui-Pi-Tang & $581(2.5)$ & Shao-Fu-Zhu-Yu-Tang & $1286(2.8)$ \\
\hline & Xiang-Sha-Liu-Jun-Zi-Tang & $580(2.5)$ & Qiong-Gui-Jiao-Ai-Tang & $978(2.1)$ \\
\hline & Si-Wu-Tang & $495(2.1)$ & Si-Wu-Tang & 879 (1.9) \\
\hline & Qiong-Gui-Jiao-Ai-Tang & $483(2.1)$ & Bao-Chan-Wu-You-Fang & $847(1.9)$ \\
\hline & Zuo-Gui-Wan & $481(2.1)$ & Long-Dan-Xie-Gan-Tang & $833(1.8)$ \\
\hline
\end{tabular}

${ }^{a}$ the total numbers of diagnosis before and after prenatal visit were 57,941 and 111,403 , respectively

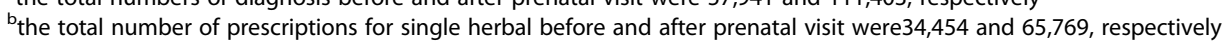

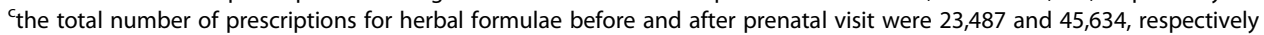

before the first prenatal visit. After the first prenatal visit, abnormal bleeding from the female genital tract (53.3\%), dysmenorrhea (9.0\%), and hypertension complicating pregnancy, childbirth, and the puerperium (7.7\%) were the three most common primary diagnoses. Before the first prenatal visit, the most frequently used single herb prescribed was Cyperi Rhizoma (Xiang $\mathrm{Fu}$ ) (5\%), followed by Cuscutae Semen ( $\mathrm{Tu} \mathrm{Si}$ $\mathrm{Zi})(4.5 \%)$ and Leonuri Herba (Yi Mu Cao) (4.2\%).
After the first prenatal visit, the top three single herbs prescribed differed from those prescribed before the first prenatal visit. Cyperi Rhizoma (Xiang Fu) (4.7\%) was the most frequently prescribed single herb, followed by Leonuri Herba (Yi Mu Cao) (4.1\%) and Corydalis Rhizoma (Yan $\mathrm{Hu} \mathrm{Su}$ ) (3.2\%). The top three most frequently prescribed herbal formulae were Dang-Guei-Shao-Yao-San, Jia-Wei-Xiao-Yao-San, and Wen-Jing-Tang, irrespective of the first prenatal visit. 
Table 4 Associating factors with the use of $\mathrm{CHM}$ during pregnancy $(n=81,873)$

\begin{tabular}{lll}
\hline Characteristics & aOR $(95 \% \mathrm{Cl})$ & $P$-value \\
\hline Age & $1.03(1.02-1.03)$ & $<0.001$ \\
Education & & \\
$\quad$ Below Junior & ref & \\
$\quad$ Senior & $1.04(0.98-1.09)$ & 0.080 \\
$\quad$ University or above & $1.16(1.09-1.23)$ & $<0.001$ \\
Residential area & & \\
$\quad$ Northern & ref & \\
$\quad$ Middle & $1.78(1.69-1.88)$ & $<0.001$ \\
$\quad$ Southern & $1.36(1.30-1.43)$ & 0.004 \\
$\quad$ Eastern & $1.14(1.04-1.26)$ & 0.001 \\
CHM use before 6 months of pregnancy & $5.69(5.46-5.93)$ & $<0.001$ \\
Asthma & $1.23(1.05-1.43)$ & 0.009 \\
Chronic renal disease & $1.33(1.02-1.73)$ & 0.034 \\
Cardiac valvular disease & $1.54(1.25-1.91)$ & $<0.001$ \\
\hline
\end{tabular}

Multiple logistic regression analysis was used to calculate adjusted odds ratio (aOR). Adjusted factors included age, education, income, residential area, CHM use before 6 months of pregnancy, asthma, chronic ischemic heart disease, chronic renal disease, congenital heart disease, preexisting diabetes mellitus, preexisting hypertension, sickle cell disease, cardiac valvular disease, placenta previa, and gestational hypertension

CHM Chinese herbal medicine

\section{Factors influencing the use of CHM during pregnancy}

A multivariate logistic regression model was used to examine the factors associated with CHM use during pregnancy (Table 4). The following factors were associated with a greater likelihood of using CHM: older pregnant women $(\mathrm{OR}=1.03,95 \% \mathrm{CI}$ : 1.02-1.03), women with a university education $(\mathrm{OR}=1.16$, 95\% CI: 1.09 1.23), those who did not live in northern Taiwan (middle Taiwan dwellers: $\mathrm{OR}=1.78,95 \%$ CI: 1.69-1.88; southern Taiwan dwellers: $\mathrm{OR}=1.36,95 \% \mathrm{CI}$ : $1.30-1.43$; eastern Taiwan dwellers $\mathrm{OR}=1.14$, 95\% CI: 1.04-1.26), and those who had pre-pregnancy experience with CHM $(\mathrm{OR}=5.69,95 \% \mathrm{CI}: 5.46-5.93)$. Women with asthma $(\mathrm{OR}=1.23$, 95\% CI: 1.05-1.43), chronic renal disease $(\mathrm{OR}=1.33$, 95\% CI: 1.02-1.73), or cardiac valvular disease $(\mathrm{OR}=1.54,95 \% \mathrm{CI}$ : $1.25-1.91)$ were also more likely to use CHM during pregnancy.

\section{Discussion}

Our research describes the CHM prescription patterns in a population of pregnant women in Taiwan and reveals discrepancies between diagnoses and CHM usage during each of the three trimesters. We found that the CHM utilization rate during pregnancy was $20.2 \%$. This rate is similar to those reported in prior prospective cohort studies [5, 18] and a study in Taiwan [7], but lower than those reported previously in Norway (39.7\%) [19] and mainland China (43.5\%) [1]. This may be because the NHIRD only contains medical records for cases diagnosed and drugs prescribed by TCM physicians. Thus, herbal medicines purchased and consumed by patients on their own were not included. As for factors related to CHM use, pregnant women with a higher level of education and larger income had greater rates of CHM usage. This may be because CHM users usually have a higher socioeconomic status and marry later in life, thus requiring menstruation regulation to increase the chance of pregnancy [5]. Additionally, we found that women treated with $\mathrm{CHM}$ or those who had chronic diseases before pregnancy preferred the use of CHM to treat their illness; this is similar to findings reported in a previous study of Chinese CHM users [20].

Among pregnant women treated with $\mathrm{CHM}$, the most common diagnoses were related to respiratory system diseases (such as acute nasopharyngitis, cough, allergic rhinitis, etc.). This is consistent with the findings of previous research [7]. Other common diagnoses included gastrointestinal diseases, pregnancy-related vomiting or bleeding, and pregnancy complicated by hypertension. Recent studies have demonstrated that TCM is also therapeutically effective not just for upper respiratory tract infections and gastrointestinal diseases, but also for bleeding during pregnancy, pregnancy-related vomiting, and pregnancy-induced hypertension [8, 21-24].

A previous study [7] reported data of diagnoses during the three trimesters, but the data were based on the systems of diseases (i.e., respiratory or circulatory system). We also reported data regarding diagnoses and prescriptions during the three trimesters, but our data were based on more accurate diagnoses of single diseases. The data presented herein indicate that TCM is increasingly being used to treat pregnancy-related complications and symptoms. The diagnoses of these common diseases are consistent with the changes in physiology and pathology that occur during pregnancy [25] and reveal which diseases may most correlate with TCM treatment during the three trimesters. Due to recent trends toward further refinement of modern medicine, obstetricians often focus solely on obstetrical disease, casting aside the other common medical conditions faced by pregnant women. TCM treatment may have clinically curative effects for symptoms or diseases presenting in pregnancy, reduce the side effects and frequency of conventional medicine use, and achieve improved health in pregnant women. A review of relevant literature shows that, when compared with conventional medicine alone, combined use of TCM and standard treatments exhibits superior results and fewer side effects [8]. Furthermore, this research reveals the proportion of diseases diagnosed among pregnant women in Taiwan and may thus be used to guide clinical practices in the country. 
Certain herbal formulae were identified as the most commonly used by pregnant women in the present study. Jia-Wei-Xiao-Yao-San can be used to treat anxiety, irritability, stress, depression, premenstrual tension, climacteric syndrome, and infertility [26-28]. In particular, the anxiolytic effects of this herbal formula are thought to act through neurosteroid synthesis [29, 30]. Xiang-Sha-Liu-Jun-Zi-Tang has long been used in clinical practice to treat gastrointestinal discomfort such as nausea and vomiting, emaciation, and anorexia. A metaanalysis found that Xiang-Sha-Liu-Jun-Zi-Tang could improve symptoms significantly more than prokinetic drugs in the treatment of functional dyspepsia [31]. Atractylodes Rhizome (Bai Zhu) works together with Scutellariae Radix (Huang Qin) to prevent miscarriage through inhibition of maternal-fetal interface immunity [32]. Past research has shown that Cortex Eucommiae (Du Zhong) contains isoflavonoids, which were reported to exhibit phytoestrogenic and androgenic properties, likely related to the optimization of sex hormone activity in the maternal body [33].

Past studies have demonstrated that physicians and pregnant women should pay particular attention to the safety and adverse effects of drugs and herbs during pregnancy [34,35]. We found that $81.7 \%$ of patients received their first CHM prescription during the first trimester. During the first prenatal visit period, abnormal bleeding from the female genital tract $(53 \%)$ and dysmenorrhea were the most common diagnoses.

Research has confirmed that some habitual abortion cases are associated with increased blood viscosity [36]. Moreover, habitual abortions may also be associated with autoimmune antibody abnormalities, especially in anti-cardiolipin antibodies (ACLs). The pathological mechanism underlying the contribution of ACLs to habitual abortion is mainly related to thrombosis in the placental blood vessels and uterine spiral arterials, fibroid necrosis, and atherosclerosis [37]. Aspirin or heparin are thus often used to inhibit platelet aggregation and anticoagulation [38].

Our study revealed that most prescriptions and medications met the pharmacopeia's use specifications, and TCM physicians used them according to their appropriate diagnostic prescriptions. However, according to the warning from the Herbal Pharmacopeia [39], leonuri Herba (Yi Mu Cao) and Shao-Fu-Zhu-Yu-Tang should be used with caution during pregnancy due to possible adverse effects such as increased contractile frequency and activity of the uterus [40]. We speculate that these herbs are likely to be used by older, highly educated women to treat infertility caused by dysmenorrhea and endometriosis disease $[41,42]$. Therefore, we recommend that TCM physicians should be cautious when prescribing CHM for pregnant women. Practitioners should avoid excess dosing and long-term use in these patients. The use and prescription patterns of $\mathrm{CHM}$ in pregnancy warrant further study and analysis.

Despite our findings, the present study also had some limitations. First, herbs purchased and used by patients themselves were not contained in the $\mathrm{NHI}$ records and thus were not included. Therefore, the frequency of TCM utilisation may have been underestimated. Second, no laboratory data or imaging findings of labour outcomes were available in this database; thus, treatment efficacy could not be determined. To establish the underlying biological mechanisms of action of these CHMs, further research should be conducted.

\section{Conclusions}

In this population-based study, we found that demographic factors and pre-existing diseases were associated with the use of CHM in pregnant women. It is worth noting that Leonuri Herba (Yi Mu Cao) and Shao-FuZhu-Yu-Tang should be used with caution in the first trimester. Further investigations should be directed to the safety and effectiveness of the use of $\mathrm{CHM}$ in pregnant women.

\section{Supplementary information}

Supplementary information accompanies this paper at https://doi.org/10. 1186/s12906-020-03032-0.

Additional file 1: Table S1. Top 10 diagnosis and prescription in CHM users during pregnancy during follow up. Table S2. The ICD-9-CM codes for maternal comorbidities.

\section{Abbreviations}

CAM: Complementary and alternative medicine; CHM: Chinese herbal medicine; TCM: Traditional Chinese medicine; NHI: National Health Insurance; ICD-9-CM: International Classification of Disease-Clinical Modification (9th revision); LHID: Longitudinal health insurance database; ORs: Odds ratios; Cls: Confidence intervals; ACLs: Anti-cardiolipin antibodies

\section{Acknowledgements}

The data of this study is obtained from the National Health Insurance Research Database provided by the Bureau of National Health Insurance, Department of Health, and managed by the Health and Welfare Statistics Application Centre, Ministry of Health and Welfare. The interpretations and conclusions contained herein do not represent those of the Bureau of National Health Insurance, Department of Health, or Ministry of Health and Welfare. We thank Editage for English language editing of the manuscript.

\section{Authors' contributions}

WCC helped to use statistical techniques to analyse study data. HCW and SHW carried out the research and wrote the first draft of the manuscript. HSS revised the manuscript. HCW guided the research project and submitted the manuscript. All authors read and approved the final manuscript.

\section{Funding}

We would like to express our sincere gratitude for the support provided for this project in the form of grants from the Taipei Tzu Chi General Hospital (TCRD-TPE-106-22). The sponsors of the study had no role in study design, data collection, data analysis, data interpretation, or writing of the report. 


\section{Availability of data and materials}

The data that support the findings of this study are available from the Bureau of National Health Insurance, Department of Health, and managed by the Health and Welfare Statistics Application Centre, Ministry of Health and Welfare but restrictions apply to the availability of these data, which were used under license for the current study, and so are not publicly available.

\section{Ethics approval and consent to participate}

This study was approved by the Institutional Review Board of Taipei Tzu Chi Hospital, Taipei, Taiwan (Protocol No.:05-W03-050).

\section{Consent for publication}

Not applicable.

\section{Competing interests}

The authors declare that they have no competing interests.

\section{Author details}

'Department of Public Health, College of Medicine, Tzu Chi University, Hualien, Taiwan. ${ }^{2}$ Department of Medical Research, Buddhist Tzu Chi General Hospital, Hualien, Taiwan. ${ }^{3}$ Department of Chinese Medicine, Buddhist Tzu Chi General Hospital, Hualien, Taiwan. ${ }^{4}$ School of Post-baccalaureate Chinese Medicine, Tzu Chi University, Hualien, Taiwan. ${ }^{5}$ Department of Chinese Medicine, Taipei Tzu Chi Hospital, The Buddhist Tzu Chi Medical Foundation, No. 289, Jianguo Rd., Xindian Dist, New Taipei City 23142, Taiwan.

Received: 3 July 2019 Accepted: 24 July 2020

Published online: 30 July 2020

\section{References}

1. Tang L, Lee AH, Binns CW, Hui YV, Yau KKW. Consumption of Chinese herbal medicines during pregnancy and postpartum: a prospective cohort study in China. Midwifery. 2016;34:205-10.

2. Kennedy DA, Lupattelli A, Koren G, Nordeng H. Herbal medicine use in pregnancy: results of a multinational study. BMC Complement Altern Med. 2013;13:355.

3. Frawley J, Adams J, Steel A, Broom A, Gallois C, Sibbritt D. Women's use and self-prescription of herbal medicine during pregnancy: an examination of 1,835 pregnant women. Womens Health Issues. 2015;25(4):396-402.

4. Ahmed M, Hwang JH, Choi S, Han D. Safety classification of herbal medicines used among pregnant women in Asian countries: a systematic review. BMC Complement Altern Med. 2017;17(1):489.

5. Chuang CH, Chang PJ, Hsieh WS, Tsai YJ, Lin SJ, Chen PC. Chinese herbal medicine use in Taiwan during pregnancy and the postpartum period: population-based cohort study. Int J Nurs Stud. 2009;46(6):787-95.

6. Ong CO, Chan LY, Yung PB, Leung TN. Use of traditional Chinese herbal medicine during pregnancy: a prospective survey. Acta Obstet Gynecol Scand. 2005:84(7):699-700.

7. Yeh HY, Chen YC, Chen FP, Chou LF, Chen TJ, Hwang SJ. Use of traditional Chinese medicine among pregnant women in Taiwan. Int J Gynaecol Obstet. 2009;107(2):147-50.

8. Li L, Leung PC, Chung TK, Wang CC. Systematic review of Chinese medicine for miscarriage during early pregnancy. Evid Based Complement Alternat Med. 2014;2014:753856.

9. Wiles J, Rosenberg MW. 'Gentle caring experience'. Seeking alternative health care in Canada. Health Place. 2001;7(3):209-24.

10. Astin JA. Why patients use alternative medicine: results of a national study. JAMA. 1998;279(19):1548-53.

11. Kemppainen LM, Kemppainen TT, Reippainen JA, Salmenniemi ST, Vuolanto $\mathrm{PH}$. Use of complementary and alternative medicine in Europe: healthrelated and sociodemographic determinants. Scand J Public Health. 2018; 46(4):448-55.

12. Chen FP, Chen TJ, Kung YY, Chen YC, Chou LF, Chen FJ, Hwang SJ. Use frequency of traditional Chinese medicine in Taiwan. BMC Health Serv Res. 2007;7:26.

13. Hsieh SC, Lai JN, Lee CF, Hu FC, Tseng WL, Wang JD. The prescribing of Chinese herbal products in Taiwan: a cross-sectional analysis of the national health insurance reimbursement database. Pharmacoepidemio Drug Saf. 2008;17(6):609-19.
14. Cheng SH, Chiang TL. The effect of universal health insurance on health care utilization in Taiwan. Results from a natural experiment. JAMA. 1997; 278(2):89-93.

15. Lin LY, Warren-Gash C, Smeeth L, Chen PC. Data resource profile: the National Health Insurance Research Database (NHIRD). Epidemiol Health. 2018:40:e2018062.

16. Hsieh CY, Su CC, Shao SC, Sung SF, Lin SJ, Kao Yang YH, Lai EC. Taiwan'S National Health Insurance Research Database: past and future. Clin Epidemiol. 2019;11:349-58.

17. Bateman BT, Mhyre JM, Hernandez-Diaz S, Huybrechts KF, Fischer MA, Creanga AA, Callaghan WM, Gagne JJ. Development of a comorbidity index for use in obstetric patients. Obstet Gynecol. 2013;122(5):957-65.

18. Chuang CH, Hsieh WS, Guo YL, Tsai YJ, Chang PJ, Lin SJ, Chen PC. Chinese herbal medicines used in pregnancy: a population-based survey in Taiwan. Pharmacoepidemio Drug Saf. 2007;16(4):464-8.

19. Nordeng H, Bayne K, Havnen GC, Paulsen BS. Use of herbal drugs during pregnancy among 600 Norwegian women in relation to concurrent use of conventional drugs and pregnancy outcome. Complement Ther Clin Pract. 2011;17(3):147-51.

20. Zhu X, Qi X, Hao J, Huang Z, Zhang Z, Xing X, Cheng D, Xiao L, Xu Y, Zhu P, et al. Pattern of drug use during the first trimester among Chinese women: data from a population-based cohort study. Eur J Clin Pharmacol. 2010; 66(5):511-8.

21. Hollyer $\mathrm{T}$, Boon $\mathrm{H}$, Georgousis A, Smith M, Einarson A. The use of CAM by women suffering from nausea and vomiting during pregnancy. BMC Complement Altern Med. 2002;2:5.

22. Thomson M, Corbin R, Leung L. Effects of ginger for nausea and vomiting in early pregnancy: a meta-analysis. J Am Board Fam Med. 2014;27(1):115-22.

23. Wang C, Wang H, Liu X, Xu D, Tang Y, Luo P. Traditional Chinese medicine for the treatment of influenza: a systematic review and meta-analysis of randomized controlled trials. J Tradit Chin Med. 2014;34(5):527-31.

24. Chu MHK, Wu IXY, Ho RST, Wong CHL, Zhang AL, Zhang Y, Wu JCY, Chung $\mathrm{VCH}$. Chinese herbal medicine for functional dyspepsia: systematic review of systematic reviews. Ther Adv Gastroenterol. 2018:11:1756284818785573.

25. Soma-Pillay P, Nelson-Piercy C, Tolppanen H, Mebazaa A. Physiological changes in pregnancy. Cardiovas J Afr. 2016;27(2):89-94.

26. Washio M. Kami-shoyo-san is usually used for women. Psychiatry Clin Neurosci. 2003:57(3):345

27. Park DM, Kim SH, Park YC, Kang WC, Lee SR, Jung IC. The comparative clinical study of efficacy of Gamisoyo-San (Jiaweixiaoyaosan) on generalized anxiety disorder according to differently manufactured preparations: multicenter, randomized, double blind, placebo controlled trial. J Ethnopharmacol. 2014;158(Pt A):11-7.

28. Park SW, Kim YK, Lee JG, Kim SH, Kim JM, Yoon JS, Park YK, Lee YK, Kim YH. Antidepressant-like effects of the traditional Chinese medicine kami-shoyosan in rats. Psychiatry Clin Neurosci. 2007;61(4):401-6.

29. Mizowaki M, Toriizuka K, Hanawa T. Anxiolytic effect of Kami-Shoyo-San (TJ-24) in mice: possible mediation of neurosteroid synthesis. Life Sci. 2001;69(18):2167-77.

30. Cao GP, Gui D, Fu LD, Guo ZK, Fu WJ. Anxiolytic and neuroprotective effects of the traditional Chinese medicinal formulation Dan-zhi-xiao-yao-san in a rat model of chronic stress. Mol Med Rep. 2016;14(2):1247-54.

31. Xiao Y, Liu YY, Yu KQ, Ouyang MZ, Luo R, Zhao XS. Chinese herbal medicine liu jun zi tang and xiang sha liu jun zi tang for functional dyspepsia: metaanalysis of randomized controlled trials. Evid Based Complement Alternat Med. 2012;2012:936459.

32. Zhong XH, Zhou ZX, Li TS, Wang EQ, Shi WY, Chu SM. Anti-abortive effect of Radix scutellariae and Rhizoma atractylodis in mice. Am J Chin Med. 2002:30(1):109-17.

33. Hussain T, Tan B, Liu G, Oladele OA, Rahu N, Tossou MC, Yin Y. Healthpromoting properties of Eucommia ulmoides: a review. Evid Based Complement Alternat Med. 2016;2016:5202908.

34. Crespin S, Bourrel R, Hurault-Delarue C, Lapeyre-Mestre M, Montastruc JL, Damase-Michel C. Drug prescribing before and during pregnancy in south West France: a retrolective study. Drug Saf. 2011;34(7):595-604.

35. Kao LT, Chen YH, Lin HC, Chung SD. Prescriptions for category D and X drugs during pregnancy in Taiwan: a population-based study. Pharmacoepidemiol Drug Saf. 2014;23(10):1029-34.

36. Pritchard AM, Hendrix PW, Paidas MJ. Hereditary thrombophilia and recurrent pregnancy loss. Clin Obstet Gynecol. 2016;59(3):487-97.

37. Santos TDS, leque AL, de Carvalho HC, Sell AM, Lonardoni MVC, Demarchi IG, de Lima Neto QA, Teixeira JJV. Antiphospholipid syndrome and recurrent 
miscarriage: a systematic review and meta-analysis. J Reprod Immunol. 2017:123:78-87.

38. Ziakas PD, Pavlou M, Voulgarelis M. Heparin treatment in antiphospholipid syndrome with recurrent pregnancy loss: a systematic review and metaanalysis. Obstet Gynecol. 2010;115(6):1256-62.

39. Taiwan Herbal Pharmacopeia 3rd Edition Committee. Taiwan Herbal Pharmacopeia 3rd Edition English version. Taiwan, R.O.C: Ministry Health and Welfare; 2019

40. Zhu YZ, Wu W, Zhu Q, Liu X. Discovery of Leonuri and therapeutical applications: from bench to bedside. Pharmacol Ther. 2018;188:26-35.

41. Lee H, Choi TY, Myung CS, Lee JA, Lee MS. Herbal medicine (Shaofu Zhuyu decoction) for treating primary dysmenorrhea: a systematic review of randomized clinical trials. Maturitas. 2016:86:64-73.

42. Zhu G, Jiang C, Yan X, Zhao S, Xu D, Cao Y. Shaofu Zhuyu decoction regresses Endometriotic lesions in a rat model. Evid Based Complement Alternat Med. 2018;2018:3927096.

\section{Publisher's Note}

Springer Nature remains neutral with regard to jurisdictional claims in published maps and institutional affiliations.

Ready to submit your research? Choose BMC and benefit from:

- fast, convenient online submission

- thorough peer review by experienced researchers in your field

- rapid publication on acceptance

- support for research data, including large and complex data types

- gold Open Access which fosters wider collaboration and increased citations

- maximum visibility for your research: over $100 \mathrm{M}$ website views per year

At BMC, research is always in progress.

Learn more biomedcentral.com/submissions 